\title{
The relevance of MRI for predicting neurological recovery following cervical traumatic spinal cord injury
}

\author{
Joanie Martineau ${ }^{1,2} \cdot$ Julien Goulet ${ }^{1,2} \cdot$ Andréane Richard-Denis $\mathbb{D}^{1,2} \cdot$ Jean-Marc Mac-Thiong ${ }^{1,2}$
}

Received: 4 December 2018 / Revised: 24 April 2019 / Accepted: 24 April 2019 / Published online: 23 May 2019

(C) International Spinal Cord Society 2019

\begin{abstract}
Study design Retrospective cohort study of 82 patients with cervical traumatic spinal cord injury (TSCI).

Objectives Determine the relevance of preoperative MRI to predict neurological recovery following cervical TSCI.

Setting Level I trauma center specialized in TSCI.

Methods The following three MRI parameters were assessed: presence of an intramedullary hemorrhage, intramedullary lesion length and maximal compression of the spinal cord compression (MSCC). Analyses were performed to assess the relationship between MRI parameters and three neurological outcomes: ASIA motor score (AMS), improvement by at least one ASIA impairment scale (AIS) grade (conversion of AIS grade), and reaching AIS grade D or E.

Results Predicting AMS based on initial AIS grade and intramedullary hemorrhage resulted in a validation R-squared of 0.662 , and of 0.636 when using only the initial AIS grade. Predicting conversion of AIS grade based on initial AIS grade, intramedullary hemorrhage and lesion length resulted in a validation c-index of 0.704 , and of 0.727 when using only the initial AIS grade. Predicting the likelihood of a follow-up AIS grade D or E based on initial AIS grade and intramedullary hemorrhage in a validation c-index of 0.903 , and of 0.873 when using only the initial AIS grade.

Conclusions Intramedullary hemorrhage and lesion length assessed from preoperative MRI were predictors of the neurological recovery following cervical TSCI. However, the clinical benefit of these MRI parameters to predict the neurological recovery remains limited when the initial AIS grade is available, confirming that the initial neurological status remains the most important predictor of the neurological outcome.
\end{abstract}

\section{Introduction}

Traumatic spinal cord injuries (TSCI) can cause a wide range of deficits, but most patients will remain with significant neurological deficits. Predicting the long-term outcome is important for caregivers to provide better counseling to patients about future recovery and return to normal activities, and to select appropriate treatment and rehabilitation needs. However, it remains difficult to

Supplementary information The online version of this article (https:// doi.org/10.1038/s41393-019-0295-z) contains supplementary material, which is available to authorized users.

Jean-Marc Mac-Thiong

jean-marc.mac-thiong@umontreal.ca

1 Faculty of Medicine, Université de Montréal, Montréal, QC, Canada

2 Hôpital du Sacré-Coeur de Montréal, Montréal, QC, Canada determine the long-term neurological recovery after TSCI although several predictors have been identified [1,2].

There is a growing interest for correlating magnetic resonance imaging (MRI) findings to initial injury severity and neurological recovery [3-8]. Previous studies suggest that the extrinsic compression of the spinal cord seen from MRI is associated with the initial severity in cervical and thoracolumbar TSCI [5-11] but the relationship with neurological recovery is equivocal. Multivariable regression analyses failed to demonstrate a significant association between measurements of extrinsic spinal cord compression and neurological recovery, especially when accounting for the initial neurological status [8, 11]. On the opposite, various studies [3, 4, 6-10] suggest that intramedullary signal abnormalities seen on MRI initially after the TSCI could be associated with the neurological recovery. While many studies were performed on a limited number of patients $(\mathrm{n}<40)[3,4,7]$, other with larger cohorts [9, 10] failed to provide statistical analyses to assess the association between intramedullary MRI findings and neurological 
recovery. Using multivariable regression analyses, Miyanji et al. [8] reported a significant relationship between followup motor score and the presence of spinal cord hemorrhage. However, the short length of follow-up for some patients and the wide variability (between 1 and 35 months after the TSCI), as well as the inclusion of 22 patients with a normal baseline neurological status limit their conclusions. Gupta et al. [6] also observed an association between follow-up motor score and spinal cord hemorrhage but the follow-up was short (between 3 and 6 months after the TSCI). Furthermore, they suggest that the length of the signal abnormality measured from $\mathrm{T} 2 \mathrm{~W}$ images could be a predictor of neurological recovery since it almost reached statistical significance in their multivariable regression model of follow-up motor score. Wilson et al. [12] found that the initial presence of spinal cord edema and hemorrhage was related to deteriorating functional status 6 to 12 months after the TSCI. Unfortunately, they did not evaluate if a similar association was present with neurological recovery.

In order to clarify the relevance of MRI for predicting the neurological recovery in TSCI patients, this paper aims to determine the association between three MRI parameters and neurological recovery 6 to 12 months after a cervical TSCI. More specifically, we will perform univariate and multivariable analyses to identify whether the presence of intramedullary hemorrhage, the length of the intramedullary signal abnormality (lesion length), and maximal spinal cord compression (MSCC) are predictive of neurological recovery 6 to 12 months following cervical TSCI.

\section{Methods}

\section{Cohort description}

We performed a retrospective analysis of a prospective cohort of 82 patients managed at a single Level I trauma center specialized in TSCI between 2010 and 2017. The inclusion criteria were the following: (1) age of 16 years or older, (2) presence of a cervical TSCI with a neurological level of injury (NLI) from $\mathrm{C} 0$ to $\mathrm{C} 8$, (3) spinal surgery performed within 5 days of the trauma, (4) baseline preoperative MRI of the spine performed within $72 \mathrm{~h}$ of the trauma, (5) initial neurological examination within $72 \mathrm{~h}$ of the trauma and prior to surgery, (6) surgery performed within 5 days after the TSCI, and (7) follow-up neurological examination between 6 and 12 months after the TSCI. Exclusion criteria consisted of: (1) absence of neurological impairment at admission to our institution, (2) no surgery or late surgery performed more than 5 days after the TSCI, (3) no MRI or MRI performed $>72 \mathrm{~h}$ after the TSCI, or (4) neurological deficit prior to TSCI.

\section{Dependent variables}

Neurological examination was performed within $72 \mathrm{~h}$ of the trauma and at follow-up between 6 and 12 months after the TSCI, according to the International standards for neurological classification of spinal cord injury (ISNCSCI). The severity of the neurological injury was determined using the American Spinal Injury Association (ASIA) impairment scale (AIS), from grade A-E: (A) complete, (B) sensory incomplete, (C) motor incomplete with less than half of key muscles below the NLI with anti-gravity strength, (D) motor incomplete with at least half of key muscles below the NLI with anti-gravity strength, and (E) normal. The ASIA motor score (AMS) out of 100 was also calculated for each patient based on the rating of each key muscle bilaterally for myotomes from $\mathrm{C} 5$ to $\mathrm{T} 1$ and $\mathrm{L} 2$ to $\mathrm{S} 1$.

The analyses were performed using four different outcomes (dependent variables) derived from the ISNCSCI examination and depicting the neurological status at followup 6-12 months after the TSCI: (1) AIS grade A, B, C, D or E (ordinal), (2) AMS (continuous), (3) conversion of AIS grade defined as the presence or absence of improvement by at least one AIS grade (binary), and (4) achieving or not achieving AIS grade D or E (binary). The baseline and follow-up AIS grade were available for all 82 patients. The follow-up AMS could be calculated only for 73 patients because joint contractures present during follow-up precluded valid testing of all key muscles in 9 patients. The last two binary outcomes were applied only to the subset of 45 patients with initial AIS grade A, B or C injury.

\section{Independent variables}

All preoperative MRI were performed on a 1.5 Tesla MRI scanner (Magnetom Avanto, Siemens Healthineers, Erlangen, Germany) using a standardized protocol consisting of sagittal T2 TSE, sagittal T1 TSE, sagittal STIR, sagittal 2D MEDIC, axial 2D MEDIC and axial T2 TSE sequences. The following three MRI parameters were analyzed as potential predictors of the neurological outcome: (1) presence or absence of intramedullary hemorrhage (binary), (2) lesion length (continuous), and (3) MSCC (continuous). A single observer (medical student) performed all radiological measurements with the training and supervision from a senior orthopedic resident and a spine surgeon experienced in interpreting MRI for TSCI. All measurements were reviewed by the spine surgeon. The presence of intramedullary hemorrhage was diagnosed from a hypointense signal on $\mathrm{T} 2$ images with a thin rim of hyperintensity [6-8]. Lesion length was measured in millimeters as the maximum cranio-caudal length of signal abnormality on sagittal T2 images, which includes signals from cord edema, contusion and hemorrhage [6, 7]. MSCC was calculated from 
mid-sagittal T2 weighted images, and refers to the percentage decrease in sagittal diameter of the spinal cord at the level of maximum spinal cord compression with respect to the expected sagittal diameter of the spinal cord (mean value of the sagittal diameter measured at the uninjured levels directly above and below the level of maximum spinal cord compression) [13]. More specifically, we used the following formula:

$M S C C=1-\left(\frac{d_{\mathrm{i}}}{\left(d_{\mathrm{a}}+d_{\mathrm{b}}\right) / 2}\right) \times 100 \%$,

where $d_{\mathrm{i}}$ refers to the antero-posterior spinal cord diameter at the level of maximum compression, $d_{\mathrm{a}}$ is the anteroposterior spinal cord diameter at the nearest normal level above the site of injury, and $d_{\mathrm{b}}$ is the antero-posterior spinal cord diameter at the nearest normal level below the site of injury. Previous studies [13, 14] have confirmed that the reproducibility of MSCC is sufficient for clinical and research use.

\section{Statistical analyses}

Following descriptive statistics, we conducted univariate analyses to determine if there were associations between the three MRI parameters and four neurological outcomes. $X^{2}$ tests were done to evaluate the association between intramedullary hemorrhage and AIS grade at follow-up. Mean AMS was compared between groups with and without intramedullary hemorrhage using a Wilcoxon-Mann-Whitney's test. A Fisher exact test was used to determine the association between intramedullary hemorrhage and conversion of AIS grade or achievement of AIS grade of D or E at follow-up. Analysis of variance (ANOVA) was then used to assess if lesion length and MSCC were different according to follow-up AIS grade. In the presence of a statistical different with the ANOVA, post-hoc tests using Fisher's Least Significant Difference (LSD) were performed to identify pairwise differences between AIS grades. Correlations between lesion length, MSCC and follow-up AMS were assessed using Pearson's correlation coefficients. Lesion length and MSCC were compared based on the presence or absence of conversion of AIS grade at followup using Wilcoxon-Mann-Whitney's tests. Similarly, lesion length and MSCC were compared based on the achievement of AIS grade of D or E at follow-up using Wilcoxon-MannWhitney's tests. A level of significance of 0.05 was used for all statistical tests.

Univariates analyses were used to determine the main candidate predictors (from the three MRI parameters) to include in the multivariable prediction models. On the basis of our clinical experience and on the literature [1,2], the following co-variables were also included in the multivariable analyses as potential predictors: sex, age, initial
AIS grade and AMS at admission, delay between trauma and surgery, and injury severity score (ISS).

Prediction models were derived from the LASSO (Least Absolute Shrinkage and Selection Operator) method using the R Project for Statistical Computing. LASSO algorithms analyze subsets of independent variables (candidate predictors) - rather than analyzing individually each variableto find the best subgroups of independent variables associated with the dependent variable. The independent variables involved in the LASSO method consisted of the MRI parameters significantly associated with each outcome from univariate analyses as well as all co-variables.

The LASSO method selects the best subset of predictors by minimizing the sum of the error of fit test and the norm of the predictors. Accordingly, adding a predictor is penalizing: if a predictor is added, it means that the method is removing other redundant variables to return the best smallest subset of predictors. It has been shown to reduce overfitting and remove redundant variables [14]. The prediction models were computed with IBM SPSS Statistics 25 software. Multiple linear regression was performed to obtain a model predicting follow-up AMS, while logistic regression was used to obtain models predicting conversion of AIS grade and achievement of AIS grade D or E at follow-up. We performed 10-fold cross-validation to validate the prediction models, and calculated the $R^{2}$ (for linear regression model) or c-index (for logistic regression models) on the validation dataset to assess the predictive performance of the models. The c-index was determined from the area under the ROC (receiver operating characteristic) curve (AUC).

In order to further evaluate the relevance of preoperative MRI parameters to predict neurological recovery, we have also assessed the predictive performance of using only the initial AIS grade (without the MRI parameters) for predicting the outcomes using logistic and linear regression analyses.

\section{Results}

\section{Characteristics of patients}

Table 1 presents the baseline characteristics of the patients included in the analyses. Table 2 presents the AIS grade at admission and follow-up after surgery. Half of the entire cohort underwent conversion of the AIS grade at final follow-up, with $57 \%$ for AIS grade A, $56 \%$ for AIS grade B, $93 \%$ for AIS grade C and $22 \%$ for AIS grade D. A total of $49 \%(22 / 45)$ of patients with initial AIS grade A, B or C patients reached a follow-up AIS grade of D or E. Followup AMS $(p=0.39)$, conversion of the AIS grade $(p=0.19)$ and the likelihood of reaching AIS grade D or E $(p=0.14)$ 
Table 1 Baseline demographic, injury-related and clinical characteristics of patients

\begin{tabular}{|c|c|c|c|}
\hline & & All patients & $\begin{array}{l}\text { Patients } \\
\text { with initial } \\
\text { AIS grade } \\
\text { A, B or C }\end{array}$ \\
\hline \multicolumn{2}{|l|}{$N$} & 82 & 45 \\
\hline \multicolumn{2}{|l|}{ Sex (males:females) } & $67: 15$ & $34: 11$ \\
\hline \multicolumn{2}{|c|}{ Age (years) ( \pm standard deviation) } & $52.1 \pm 16.9$ & $50.0 \pm 18.5$ \\
\hline \multicolumn{2}{|c|}{$\begin{array}{l}\text { Injury severity score (ISS) ( } \pm \text { standard } \\
\text { deviation) }\end{array}$} & $22.4 \pm 11.3$ & $25.2 \pm 13.7$ \\
\hline \multirow[t]{5}{*}{ Trauma Mechanism } & Sport & $12(14.6 \%)$ & $5(11.1 \%)$ \\
\hline & Blunt assault & $4(4.9 \%)$ & $2(4.4 \%)$ \\
\hline & Fall & $40(48.8 \%)$ & $23(51.1 \%)$ \\
\hline & Transportation & $25(30.5 \%)$ & $15(33.3 \%)$ \\
\hline & Other & $1(1.2 \%)$ & 0 \\
\hline \multirow[t]{2}{*}{$\begin{array}{l}\text { Level of } \\
\text { neurological injury }\end{array}$} & $\begin{array}{l}\text { High cervical } \\
(\mathrm{C} 0-\mathrm{C} 4)\end{array}$ & $38(46.3 \%)$ & $19(42.2 \%)$ \\
\hline & $\begin{array}{l}\text { Low } \\
\text { cervical (C5-8) }\end{array}$ & $44(53.7 \% \%)$ & $26(57.8 \%)$ \\
\hline \multicolumn{2}{|c|}{$\begin{array}{l}\text { Surgery delay (hours) }( \pm \text { standard } \\
\text { deviation) }\end{array}$} & $37.7 \pm 24.7$ & $30.4 \pm 20.5$ \\
\hline \multicolumn{2}{|c|}{ MRI delay (hours) ( \pm standard deviation) } & $14.7 \pm 10.7$ & $11.7 \pm 10.7$ \\
\hline
\end{tabular}

AIS ASIA impairment scale, MRI magnetic resonance imaging

Table 2 Distribution of patients according to initial and follow-up AIS grade $(N=82)$

\begin{tabular}{llllllll}
\hline & & \multicolumn{3}{c}{ Follow-up AIS grade } & & \multirow{2}{*}{ Total } \\
\cline { 3 - 6 } & & $\mathrm{A}$ & $\mathrm{B}$ & $\mathrm{C}$ & $\mathrm{D}$ & $\mathrm{E}$ & \\
\hline Initial AIS grade & $\mathrm{A}$ & 9 & 4 & 5 & 3 & 0 & 21 \\
& $\mathrm{~B}$ & 0 & 2 & 2 & 5 & 0 & 9 \\
& $\mathrm{C}$ & 0 & 0 & 1 & 14 & 0 & 15 \\
& $\mathrm{D}$ & 0 & 0 & 1 & 28 & 8 & 37 \\
& Total & 9 & 6 & 9 & 50 & 8 & 82 \\
\hline
\end{tabular}

AIS ASIA impairment scale

were not different between patients with low cervical C0-C4 and high cervical C5-C8 neurological level of injury.

\section{Univariate analyses}

Table 3 shows that the distribution of patients with hemorrhage is not uniformed $\left(p<10^{-7}\right)$ but skewed to the left with respect to the follow-up AIS grade. The proportion of patients presenting intramedullary hemorrhage significantly increases with more severe follow-up AIS grade. Table 4 shows that patients presenting with intramedullary hemorrhage have a significantly lower AMS at follow-up $(p<0.0005)$. Table 5 shows that patients presenting an intramedullary hemorrhage are less likely to undergo conversion of the AIS grade $(p=0.045)$ and to reach a follow-up AIS grade D or E $(p=0.008)$.

Table 3 indicates that decreasing lesion length is observed with less severe AIS grade at follow-up ( $p=$ 0.004). Post-hoc tests showed significant differences in lesion length between patients with AIS grade A vs. C, A vs. D, A vs. E. As presented in Table 4, increased lesion length is correlated to decreased follow-up AMS ( $r=$ $-0.463, p=0.00004)$. Table 5 indicates that the lesion length is significantly smaller for patients undergoing AIS grade conversion $(p=0.05)$. However, the lesion length was similar for patients reaching or not reaching follow-up AIS grade D or E when their initial AIS grade was A, B or C.

Univariate analyses presented in Tables 3, 4 and 5 are not consistent with an association between MSCC and neurological outcome, except for an isolated statistically significant correlation with follow-up AMS $(r=-0.271$, $p=0.02$ ).

\section{Multivariable analyses}

On the basis of the results from the univariate analyses, the following two MRI parameters were included as candidate predictors in the multivariable analyses to predict the neurological outcome: (1) the absence or presence of intramedullary hemorrhage and (2) lesion length.

Table 6 (Supplementary file) presents the model for predicting follow-up AMS. Initial AIS grade and the presence of intramedullary hemorrhage were predictive of follow-up AMS, and $74.0 \%$ of the variance was explained by the generated prediction model $\left(R^{2}=0.740\right)$. The performance of the prediction model following 10-fold crossvalidation revealed an out-of-sample R-squared of 0.662 (95\% CI [0.610-0.714]) on the validation dataset, reflecting that $66.2 \%$ of the variance of predicted follow-up AMS is explained by the model. When considering only the initial AIS grade in the analysis, the resulting $R^{2}$ was 0.704 , with an out-of-sample $R^{2}$ of 0.636 (95\% CI [0.578-0.694]) on the validation dataset.

Table 7 (Supplementary file) presents the model for predicting conversion of AIS grade in patients with initial AIS grade A, B or C. Initial AIS grade, the presence of intramedullary hemorrhage and lesion length were significant predictors of conversion of AIS grade. The c-index associated with the development dataset used to generate the model was 0.770 . With 10 -fold crossvalidation, the out-of-sample c-index calculated using the validation dataset was 0.704 (95\% CI [0.588-0.820]). When considering only the initial AIS grade in the analysis, the resulting c-index was 0.720 , with an out-ofsample c-index of 0.727 (95\% CI [0.542-0.913]) on the validation dataset. 
Table 3 Comparisons between MRI parameters and follow-up AIS grade $(N=82)$

\begin{tabular}{|c|c|c|c|c|c|c|}
\hline & \multicolumn{5}{|c|}{ Follow-up AIS grade } & \multirow[t]{2}{*}{$p$-value } \\
\hline & A & $\mathrm{B}$ & $\mathrm{C}$ & $\mathrm{D}$ & $\mathrm{E}$ & \\
\hline Intramedullary hemorrhage ( $\%$ patients) & $66.7 \%$ & $66.7 \%$ & $33.3 \%$ & $4 \%$ & $0 \%$ & $<10^{-7}$ \\
\hline Lesion length $(\mathrm{mm})$ ( \pm standard deviation) & $51.9 \pm 27.3^{\mathrm{a}, \mathrm{b}, \mathrm{c}}$ & $37.3 \pm 8.2865$ & $28.9 \pm 11.7^{\mathrm{a}}$ & $27.5 \pm 16.2^{\mathrm{b}}$ & $24.5 \pm 22.1^{\mathrm{c}}$ & 0.004 \\
\hline MSCC $(\%)( \pm$ standard deviation $)$ & $27.5 \pm 33.4$ & $27.7 \pm 14.7$ & $15.9 \pm 14.1$ & $17.2 \pm 15.5$ & $17.2 \pm 8.7$ & 0.360 \\
\hline
\end{tabular}

$P$ values for post-hoc LSD tests: $\mathrm{a}=0.007 ; \mathrm{b}=0.0002 ; \mathrm{c}=0.002$

AIS ASIA impairment scale, MSCC maximal spinal cord compression

Table 4 Comparisons between MRI parameters and follow-up AMS $(N=82)$

\begin{tabular}{llll}
\hline & & Follow-up AMS ( \pm standard deviation) & $p$-value \\
\hline Intramedullary & Absence & $82.3 \pm 26.7$ & $<0.0005$ \\
hemorrhage & Presence & $29.5 \pm 26.4$ & $p$-value \\
& & Correlation coefficient with follow-up AMS & 0.00004 \\
Lesion length & & -0.463 & 0.020 \\
MSCC & & -0.271 & \\
\hline
\end{tabular}

AMS ASIA motor score, $M S C C$ maximal spinal cord compression

Table 5 Comparison between MRI parameters and improvement in AIS grade for patients with an initial AIS grade A, B or C

\begin{tabular}{|c|c|c|c|c|c|c|}
\hline & \multicolumn{3}{|c|}{ Conversion of AIS grade } & \multicolumn{3}{|c|}{ Follow-up AIS grade D or E } \\
\hline & No & Yes & $p$-value & No & Yes & $p$-value \\
\hline $\mathrm{N}$ & 12 & 33 & & 23 & 22 & \\
\hline Intramedullary hemorrhage (\% patients) & $58 \%$ & $24 \%$ & 0.039 & $56 \%$ & $9 \%$ & 0.008 \\
\hline Lesion length $(\mathrm{mm})$ ( \pm standard deviation) & $46.9 \pm 25.7$ & $34.6 \pm 13.14$ & 0.049 & $40.5 \pm 20.7$ & $35.2 \pm 14.5$ & 0.296 \\
\hline MSCC (\%) ( \pm standard deviation) & $22.5 \pm 30.1$ & $20.6 \pm 16.3$ & 0.713 & $23.3 \pm 23.7$ & $18.9 \pm 16.8$ & 0.683 \\
\hline
\end{tabular}

AIS ASIA impairment scale, MSCC maximal spinal cord compression

Table 8 (Supplementary file) presents the model for predicting a follow-up AIS grade D or E in patients with initial AIS grade A, B or C. Initial AIS grade and the presence of intramedullary hemorrhage were significant predictors of conversion of AIS grade. The c-index associated with the development dataset used to generate the model was 0.899 . With 10 -fold cross-validation, the out-of-sample c-index calculated using the validation dataset was 0.903 (95\% CI [0.785-1]). When considering only the initial AIS grade in the analysis, the resulting c-index was 0.886 , with a c-index of 0.873 (95\% CI [0.711-1]) on the validation dataset.

\section{Discussion}

Previous studies [5-11] have suggested that MRI findings were predictive of neurological recovery. Another study [12] has also proposed a prediction model of functional status using MRI findings. However, we are the first to propose validated prediction models of neurological recovery based on MRI parameters for cervical TSCI. In line with previous studies [5-11], we have confirmed that MRI parameters are significantly associated with neurological recovery 6 to 12 months following the TSCI on a statistical point of view, while controlling for co-variables such as age and initial neurological status. As opposed to Gupta et al. [6] and Miyanji et al. [8] who also performed multivariable analyses, our study only involved patients with a minimum 6 months follow-up. Such minimal followup is important when studying predictors of neurological recovery or proposing prediction models since a significant proportion of patients have not reached their full recovery before 6 months following the TSCI [15]. However, our results also suggest that the clinical benefit of the assessed MRI parameters to predict neurological recovery is relatively limited when the initial neurological status (AIS grade) is available.

Interestingly, our prediction models only used a minimal number of predictors although we initially worked with 9 
potential predictors including 3 MRI parameters. With only two predictors (initial AIS grade and intramedullary hemorrhage) for follow-up AMS and the probability to achieve AIS grade D or E, and three predictors (initial AIS grade, intramedullary hemorrhage and lesion length) for conversion of AIS grade, high predictive performance was obtained. The use of a minimal number of predictors in prediction models is highly beneficial to facilitate implementation into clinical practice. In addition, the predictive performance (R-squared and c-index) of our models is in the same range as those obtained by Gupta et al. [6] and Miyanji et al. [8] for predicting AMS, and Wilson et al. [12] for predicting functional recovery using clinical and MRI assessments, therefore supporting the validity of our analyses.

Although multiple MRI parameters have been used in the past to describe TSCI, we have limited our analyses to three MRI parameters (intramedullary hemorrhage, lesion length and MSCC) that are easy to assess and that have been proposed as potential predictors of neurological recovery in previous studies. In accordance with the literature [1, 2], our findings suggest that the initial neurological status is the most important predictor of neurological recovery. Although the presence of intramedullary hemorrhage was a significant predictor of neurological recovery, its inclusion in the prediction model only added a small increase in terms of prediction performance when compared to a model involving only the initial AIS grade. Similarly, addition of the lesion length for predicting the conversion of AIS grade did not result in an increase in prediction performance when compared to the model involving only the initial AIS grade. These findings are important because previous studies $[6,8]$ have only focused on the statistical significance of MRI parameters for predicting neurological recovery, without fully considering their added clinical value on model performance when compared to models including only the initial neurological status.

The value of MSCC for predicting neurological recovery was equivocal from previous studies [5-11]. Our results did not show any significant association between MSCC and either follow-up AMS, conversion of AIS grade or achievement of AIS grade D or E. This is in line with Miyanji et al. [8] who observed that MSCC was not predictive of follow-up AMS when taking into account the initial baseline AMS. Therefore, MSCC does not seem to be an important MRI parameter for predicting neurological recovery for two main reasons. First, MSCC is predominantly associated with the initial neurological status such that its effect becomes negligible when including the initial neurological status to predict neurological recoveryconsidering that the initial neurological status is the strongest predictor of neurological recovery. Second, MSCC depicts the residual spinal cord compression following the trauma seen on MRI, which underestimates the true spinal cord compression occurring at the moment of the trauma and causing most of the irreversible damage to the spinal cord.

On the opposite, the presence of intramedullary hemorrhage and to some extent the lesion length more closely reflect the irreversible damage to the spinal cord that will influence the extent of subsequent neurological recovery. The presence of hemorrhage results from vascular disruption during the trauma and is an important aspect of the primary injury to the spinal cord. The energy causing vascular disruption at the moment of the trauma will also directly damage the neural tissue in addition to the ongoing ischemic damage, and blood is also known to be noxious to parenchymal cells, thereby causing neuronal and axonal damage [16]. Although lesion length also reflects the spinal cord damage, its lesser importance in predicting long-term recovery can be partly explained by the following reasons. Measurement of the lesion length $[6,7]$ includes cord edema, contusion and hemorrhage altogether. As shown by Gupta et al. [6] and similar to the impact of MSCC, the presence of spinal cord edema is strongly associated with the initial neurological status such that its impact for predicting neurological recovery decreases significantly when including the initial neurological status to predict neurological recovery. In addition, spinal cord edema and hemorrhage are dynamic processes that evolve during the first 2 to 3 days after the TSCI, and their extent therefore varies over time [17-19]. All MRI acquisitions were performed within $72 \mathrm{~h}$ of the TSCI $(14.7 \pm 10.7 \mathrm{~h})$, but the variability likely influences the predictability of measuring lesion length. Although systematic MRI acquisitions at a specified time following the TSCI would be preferable on a methodological standpoint, it would however be impossible in practice such that any MRI parameter involving cord edema and/or hemorrhage will be affected by this limitation.

Another asset of our study is the use of three different outcomes to generate prediction models. While follow-up AMS is commonly used to assess neurological recovery, we also assessed specific outcomes for patients with initial AIS grade A, B or C injury because a previous study has demonstrated major difference in quality of life for this subgroup of patients when compared to AIS grade D patients [20]. Patients with AIS grade D TSCI also have higher chance of employment, ambulation, community reintegration and lower risk for re-hospitalization [21-26]. Previous studies have already recognized this issue by grouping patients with AIS grade A, B and C cervical TSCI altogether, as opposed to AIS grade D patients [24-26]. Accordingly, we defined two outcomes for this subgroup of patients (conversion of AIS grade and 
achievement of AIS grade D or E) that are likely to be clinically relevant.

\section{Limitations}

We acknowledge that other factors that could potentially influence the outcome such as neurochemical biomarkers, timing of neurological examination and type of surgery have not been included. Similarly, only three MRI parameters were assessed while other MRI parameters describing the spinal cord lesion have previously been described [3, 12]. These three parameters were selected because they have been investigated in previous studies and can easily be applied to the clinical setting. However, the predictive value of MRI could be further evaluated using additional parameters, especially with the advent of quantitative MRI to precisely assess the microstructural changes in the spinal cord.

The main limitation relates to the small numbers of patients in this study, particularly for smaller AIS B and C groups. Although our discrepancies in the number of subjects in each AIS group is representative of the actual distribution observed in TSCI populations [27, 28]. Accordingly, Phan et al. [29] have shown the difficulty to build prediction models for the skewed proportions of AIS grades observed for TSCI patients. Further study with a larger cohort allowing stratification based on the initial AIS grade should therefore be performed in the future. Similarly, further analysis of an additional cohort using the same methodology should be performed in the future to confirm the external validity of our findings.

\section{Conclusion}

This study suggests that intramedullary hemorrhage and lesion length assessed from preoperative MRI are predictors of the neurological recovery following cervical TSCI. However, the clinical benefit of using these MRI parameters to predict the neurological recovery remains limited when the initial AIS grade is available, confirming that the initial neurological status remains the most important predictor of the neurological outcome. Further work is required in order to propose new MRI parameters or sequences to improve the clinical benefits of MRI for prognosticating neurological recovery.

\section{Data Archiving}

According to our ethic board committee, public sharing of our local data is not allowed. However, a large part of our data has been archived in the Rick Hansen Spinal Cord Injury Registry. Specific request for confidential data sharing can be addressed to the corresponding author.
Acknowledgements We would like to thanks Mrs. Louisane Dupré and Geneviève LeBlanc for their assistance in collecting the data.

Funding This study was supported by the Fonds de recherche du Québec-Santé (FRQS), Programme d'Excellence en Médecine pour l'Initiation En Recherche (PREMIER), Department of the ArmyUnited States Army Medical Research Acquisition Activity, and Rick Hansen Spinal Cord Injury Registry.

Authors' contributions JM was responsible for designing the research protocol, extracting and analyzing the data, interpreting the result, and writing a draft of the manuscript. JG was responsible for designing the research protocol, interpreting the results, and reviewing the manuscript. ARD was responsible for interpreting the results and reviewing the manuscript. JMMT was responsible for designing the research protocol, interpreting the results, finalizing the manuscript, and securing funding.

\section{Compliance with ethical standards}

Statement of ethics We certify that all applicable institutional and governmental regulations concerning the ethical use of human volunteers were followed during the course of this research.

Conflict of interest The authors declare that they have no conflict of interest.

Publisher's note: Springer Nature remains neutral with regard to jurisdictional claims in published maps and institutional affiliations.

\section{References}

1. Al-Habib AF, Attabib N, Ball J, Bajammal S, Casha S, Hurlbert RJ. Clinical predictors of recovery after blunt spinal cord trauma: systematic review. J Neurotrauma. 2011;28:1431-43.

2. Wilson JR, Cadotte DW, Fehlings MG. Clinical predictors of neurological outcome, functional status, and survival after traumatic spinal cord injury: a systematic review. J Neurosurg Spine. 2012;17(1 Suppl):11-26.

3. Bozzo A, Marcoux J, Radhakrishna M, Pelletier J, Goulet B. The role of magnetic resonance imaging in the management of acute spinal cord injury. J Neurotrauma. 2011;28:1401-11.

4. Srinivas BH, Rajesh A, Purohit AK. Factors affecting outcome of acute cervical spine injury: a prospective study. Asian J Neurosurg. 2017;12:416-23.

5. Furlan JC, Kailaya-Vasan A, Aarabi B, Fehlings MG. A novel approach to quantitatively assess posttraumatic cervical spinal canal compromise and spinal cord compression: a multicenter responsiveness study. Spine. 2011;36:784-93.

6. Gupta R, Mittal P, Sandhu P, Saggar K, Gupta K. Correlation of qualitative and quantitative MRI parameters with neurological status: a prospective study on patients with spinal trauma. J Clin Diagn Res. 2014;8:RC13-7.

7. Magu S, Singh D, Yadav RK, Bala M. Evaluation of traumatic spine by magnetic resonance imaging and correlation with neurological recovery. Asian Spine J. 2015;9:748-56.

8. Miyanji F, Furlan JC, Aarabi B, Arnold PM, Fehlings MG. Acute cervical traumatic spinal cord injury: MR imaging findings correlated with neurologic outcome-prospective study with 100 consecutive patients. Radiology. 2007;243:820-7.

9. Ramon S, Dominguez R, Ramirez L, Paraira M, Olona M, Castello $\mathrm{T}$, et al. Clinical and magnetic resonance imaging correlation in acute spinal cord injury. Spinal Cord. 1997;35:664-73. 
10. Shimada K, Tokioka T. Sequential MR studies of cervical cord injury: correlation with neurological damage and clinical outcome. Spinal Cord. 1999;37:410-5.

11. Skeers P, Battistuzzo CR, Bernard S, Freeman BJC, Batchelor PE. Acute thoracolumbar spinal cord injury. Relationship of cord compression to neurological outcome. J Bone Jt Surg. 2018; 100:305-15.

12. Wilson JR, Grossman RG, Frankowski RF, Kiss A, Davis AM, Kulkarni AV, et al. A clinical prediction model for long-term functional outcome after traumatic spinal cord injury based on acute clinical and imaging factors. J Neurotrauma. 2012;29:2263-71.

13. Fehlings MG, Furlan JC, Massicotte EM, Arnold P, Aarabi B, Harrop $\mathrm{J}$, et al. Interobserver and intraobserver reliability of maximum canal compromise and spinal cord compression for evaluation of acute traumatic cervical spinal cord injury. Spine. 2006;31:1719-25.

14. Furlan JC, Fehlings MG, Massicotte EM, Aarabi B, Vaccaro AR, Bono CM, et al. A quantitative and reproducible method to assess cord compression and canal stenosis after cervical spine trauma. A study of interrater and intrarater reliability. Spine. 2007;32:2083-91.

15. Fawcett JW, Curt A, Steeves JD, Coleman WP, Tuszynski MH, Lammertse D, et al. Guidelines for the conduct of clinical trials for spinal cord injury as developed by the ICCP panel: spontaneous recovery after spinal cord injury and statistical power needed for therapeutic clinical trials. Spinal Cord. 2007;45:190-205.

16. Anwar MA, Al Shehabi TS, Eid AH. Inflammogenesis of secondary spinal cord injury. Front Cell Neurosci. 2016;10:98.

17. Cho N, Hachem LD, Fehlings MG. Spinal cord edema after spinal cord injury: from pathogenesis to management. In: Badaut J, Plesnila N, editors. Brain edema: from molecular mechanisms to clinical practice. London: Academic Press; 2017. p. 262-73.

18. Le E, Aarabi B, Hersh DS, Shanmuganathan K, Diaz C, Massetti $\mathrm{J}$, et al. Predictors of intramedullary lesion expansion rate on MR images of patients with subaxial spinal cord injury. J Neurosurg Spine. 2015;22:611-21.

19. Rutges JPHJ, Kwon BK, Heran M, Ailon T, Street JT, Dvorak MF. A prospective serial MRI study following acute traumatic cervical spinal cord injury. Eur Spine J. 2017;26:2324-32.
20. Richard-Denis A, Thompson C, Mac-Thiong JM. Quality of life in the subacute period following a cervical traumatic spinal cord injury based on the initial severity of the injury: a prospective cohort study. Spinal Cord. 2018;56:1042-50.

21. Pflaum C, McMcollister G, Strauss DJ, Shavelle RM, DeVivo MJ. Worklife after traumatic spinal cord injury. J Spinal Cord Med. 2006;29:377-86.

22. Cardenas DD, Hoffman JM, Kirshblum S, McKingley W. Etiology and incidence of rehospitalization after traumatic spinal cord injury: a multicenter analysis. Arch Phys Med Rehabil. 2004;85:1757-63.

23. Burns SP, Golding DG, Rolle WA,Jr, Graziani V, Ditunno JF Jr. Recovery of ambulation in motor-incomplete tetraplegia. Arch Phys Med Rehabil. 1997;78:1169-72.

24. Whiteneck GG, Gassaway J, Dijkers MP, Heinemann AW, Kreider SED. Relationship of patient characteristics and rehabilitation services to outcomes following spinal cord injury: the SCIRehab Project. J Spinal Cord Med. 2012;35:484-502.

25. Whiteneck GG, Gassaway J. SCIRehab uses practice-based evidence methodology to associate patient and treatment characteristics with outcomes. Arch Phys Med Rehabil. 2013;94:S67-74.

26. Whiteneck G, Tate D, Charlife S. Predicting community reintegration after spinal cord from demographic and injury characteristics. Arch Phys Med Rehabil. 1999;80:1485-91.

27. Dvorak MF, Noonan VK, Fallah N, Fisher CG, Finkelstein J, Kwon BK, et al. The influence of time from injury to surgery on motor recovery and length of hospital stay in acute traumatic spinal cord injury: an observational Canadian cohort study. J Neurotrauma. 2015;32:645-54.

28. Spiess MR, Muller RM, Rupp R, Schuld C. The EM-SCI Study Group, van Hedel HJA. Conversion in ASIA impairment scale during the first year after traumatic spinal cord injury. J Neurotrauma. 2009;26:2027-36.

29. Phan P, Budhram B, Zhang Q, Rivers CS, Noonan VK, Plashkes $\mathrm{T}$, et al. Highlighting discrepancies in walking prediction accuracy for patients with traumatic spinal cord injury: an evaluation of validated prediction models using a Canadian Multicenter Spinal Cord Injury Registry. Spine J 2018. https://doi.org/10.1016/j. spinee.2018.08.016. 